\title{
Global analysis of heme proteins elucidates the correlation between heme distortion and heme-binding pocket
}

\author{
Hiroko Kondo ${ }^{1}$, Masanori Fujii ${ }^{1}$, Takuma Tanioka ${ }^{1}$, Yusuke Kanematsu ${ }^{2}$, Takashi Yoshida ${ }^{1}$, \\ and Yu Takano ${ }^{3}$ \\ ${ }^{1}$ Kitami Institute of Technology \\ ${ }^{2}$ Hiroshima University \\ ${ }^{3}$ Hiroshima City University
}

August 10, 2021

\begin{abstract}
Heme proteins play diverse and important biological roles, from electron transfer and chemical catalysis, to oxygen transport and/or storage. Although the distortion of heme porphyrin correlates with the physical properties of heme, such as the redox potential and oxygen affinity, the relationship between heme distortion and the heme protein environment is unclear. We here tested the hypothesis that the protein environment of the heme-binding pocket determines heme distortion (conformation). We analyzed the correlations between the amino acid composition of the heme-binding pocket and the magnitude of heme distortion along 12 vibrational modes by using machine learning. A correlation was detected in the three lowest-vibrational modes. Analysis of heme distortions in nearly the same environments of the heme-binding pocket supported this notion. Our analyses indicate that the heme-binding pocket environment is a major factor impacting the distortion of heme porphyrin along the three lowest-vibrational modes. In addition, statistical analysis of the distortion of heme porphyrin revealed that the peaks of distributions of the ruffling and breathing distortions are shifted from 0 (the equilibrium structure). Both the ruffling and breathing distortions are correlated with the redox potential of heme, so that heme molecules with these distortions have a lower redox potential than planar molecules. These findings explain the structure-function relationship of heme and can inform protein function engineering.
\end{abstract}

\section{Hosted file}

manuscript.docx available at https://authorea.com/users/430028/articles/533586-globalanalysis-of-heme-proteins-elucidates-the-correlation-between-heme-distortion-and-hemebinding-pocket 


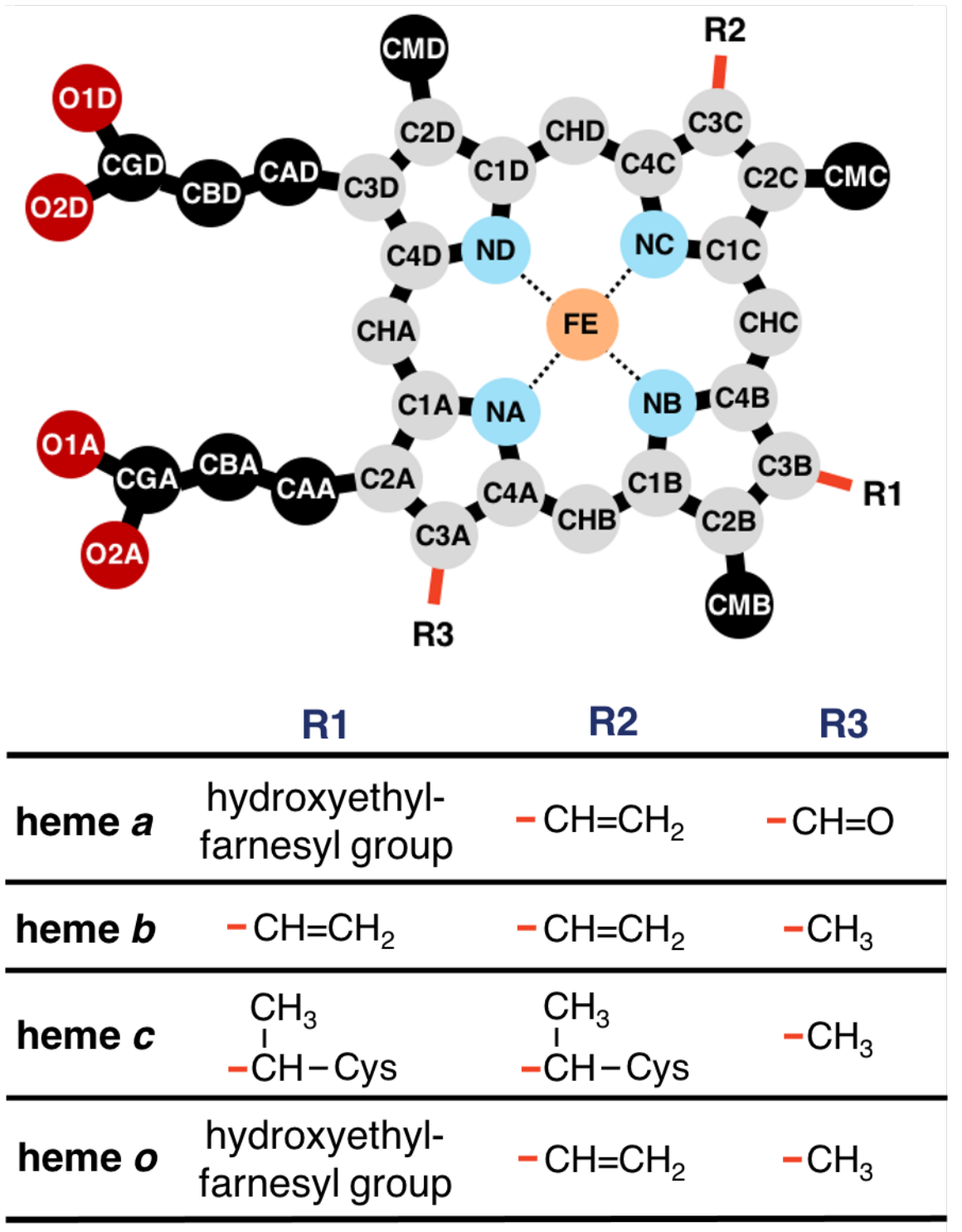



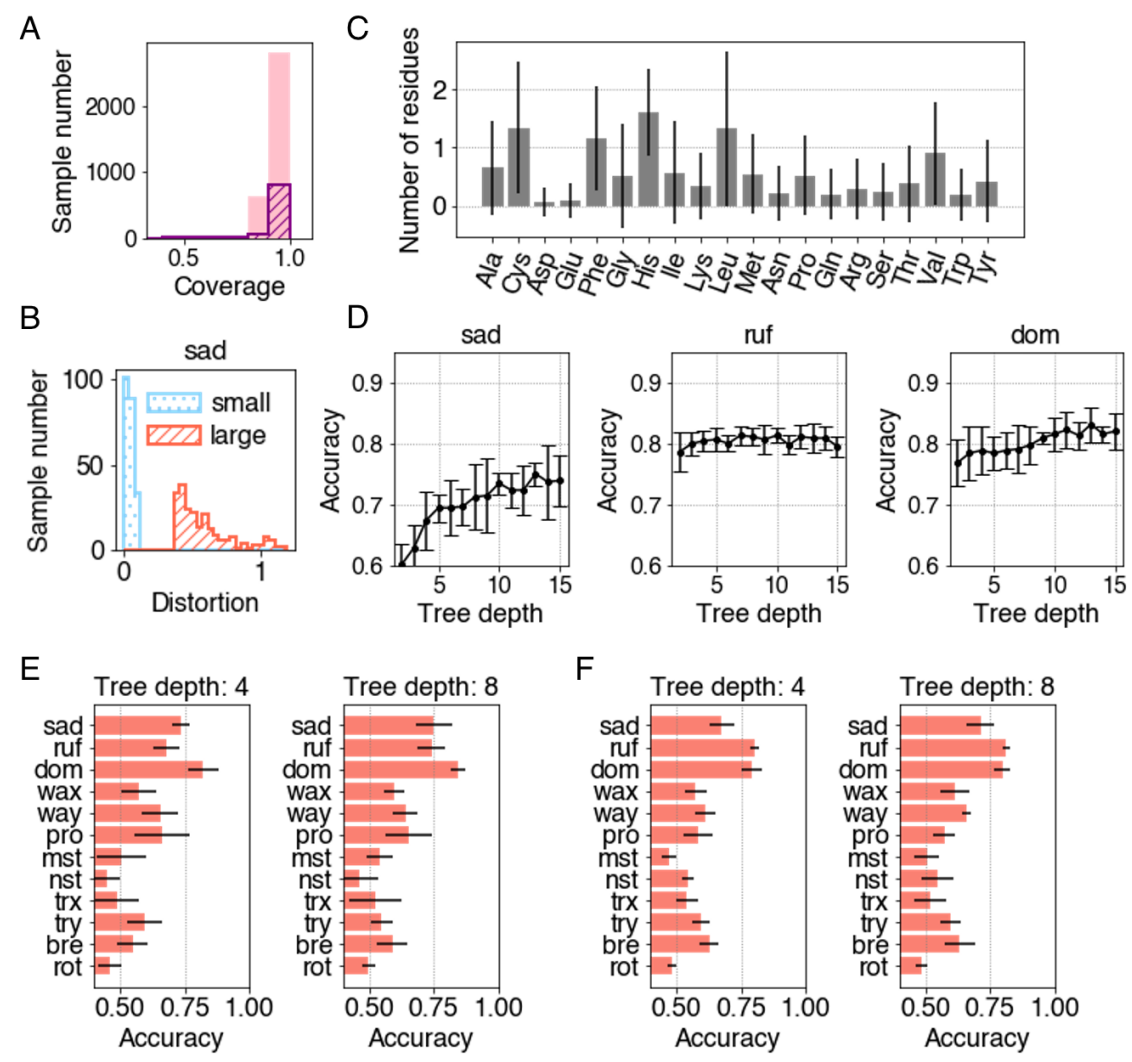

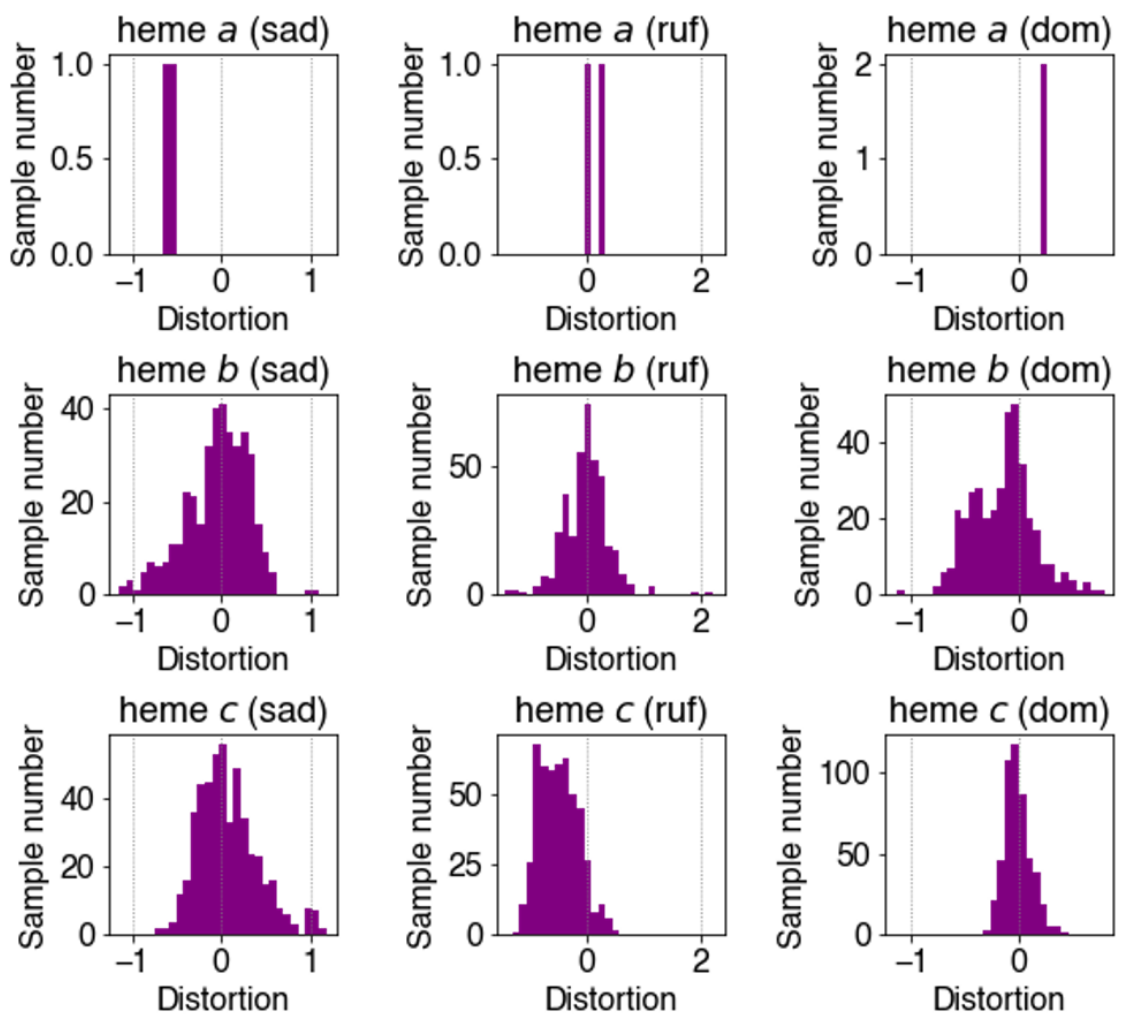

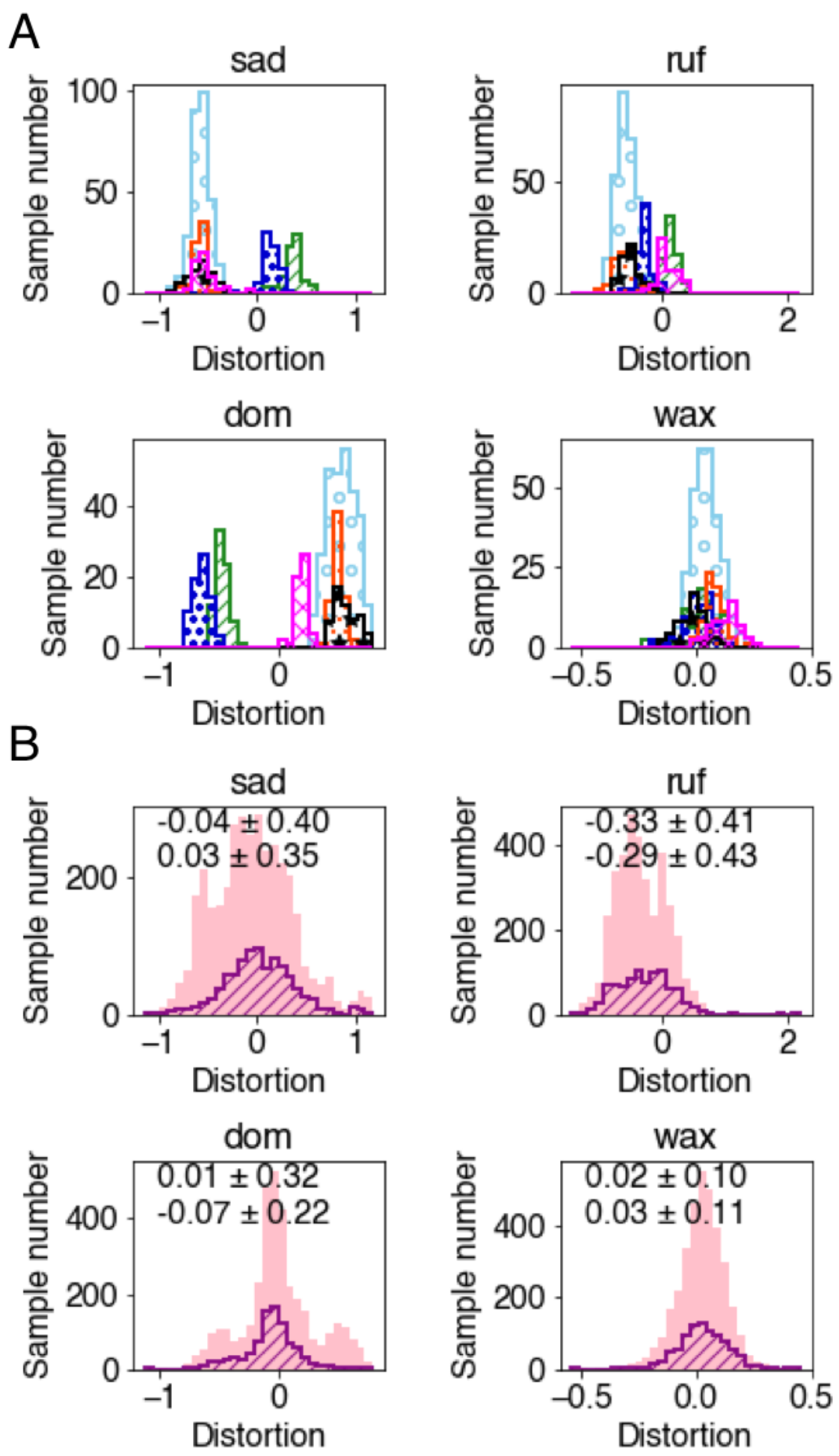

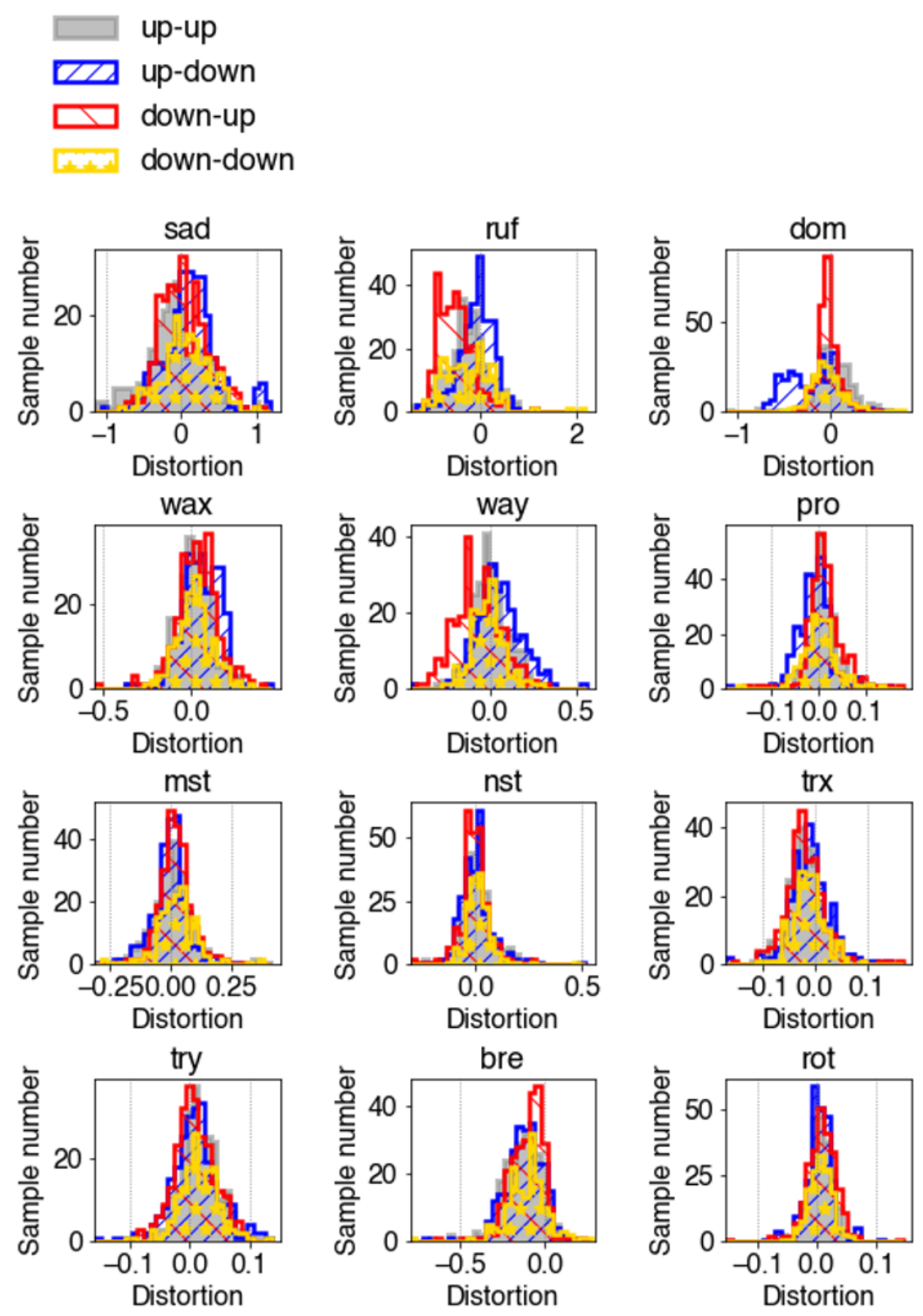